\title{
Arbeitsgestaltung für Industrie 4.0: Alte Wahrheiten, neue Herausforderungen
}

\author{
Ernst Hartmann
}

\section{Einleitung}

Das Zukunftsprojekt ,Industrie 4.0` wurde als ,strategischer Leuchtturm“ für die deutsche Innovationspolitik vorgeschlagen und entwickelt (Promotorengruppe 2013). Dies erscheint auch insofern als sinnvoll, als sich die Stimmen mehren, die in einem substanziellen Anteil industrieller Produktion an der Gesamtwirtschaftsleistung einen bedeutsamen Faktor der Innovationsfähigkeit von Volkswirtschaften sehen. Diese Sichtweise spiegelt sich etwa sehr deutlich in den Analysen der Forschungsgruppe ,Production in the Innovation Economy ‘ (PIE) des Massachusetts Institute of Technology wider (MIT; Locke und Wellhausen 2014).

Der Arbeitsgestaltung wurde in der Entwicklung des Konzepts ,Industrie 4.0` von Anfang an hohe Bedeutung beigemessen, dies zeigt sich auch deutlich im Abschlussbericht des Arbeitskreises Industrie 4.0 (Promotorengruppe 2013).

Als innovationspolitisches Zukunftsprojekt steht Industrie 4.0 somit in der Tradition deutscher Industrie- und Arbeitskultur. Vor diesem Hintergrund soll in diesem Beitrag ein Rückblick auf die Forschung und Praxis der Arbeitsgestaltung in Deutschland während der letzten fünf Jahrzehnte verbunden werden mit einen Ausblick auf neue Herausforderungen, die mit den technischen Möglichkeiten cyberphysikalischer Systeme einhergehen.

Dabei wird sich zeigen, dass viele aktuelle Fragestellungen und Gestaltungsszenarien eine lange Geschichte haben. Es könnte auch sein, dass gerade heute im Kontext der Industrie 4.0 die Zeit zur Umsetzung einiger , alter` Ideen gekommen ist. Dafür gibt es zwei Wirkkräfte: Erstens erhöht der demografische Wandel den Druck, Prinzipien wie die lernförderliche Arbeitsorganisation oder die alternsgerechte Arbeitssystemgestaltung mit wesentlich größerer Ernsthaftigkeit zu verfolgen als in der Vergangenheit. Zweitens bieten

Der Originaltext dieses Beitrags wurde überarbeitet. Das vollständige Korrekturverzeichnis finden Sie am Ende des Buchs und online unter http://dx.doi.org/10.1007/978-3-662-45915-7_16.

E. Hartmann $(\varangle)$

Institut für Innovation und Technik (iit), Berlin, Germany

e-mail: hartmann@iit-berlin.de

(C) The Author(s) 2015

A. Botthof, E.A. Hartmann (Hrsg.), Zukunft der Arbeit in Industrie 4.0,

DOI 10.1007/978-3-662-45915-7_2 
cyberphysikalische Systeme neue Möglichkeiten, etwa wenn es darum geht, komplexe Informationen zu erfassen, aufzubereiten und zu visualisieren, um sie den Nutzern vor Ort zur Verfügung zu stellen.

Das oben bereits angesprochene Konzept der , lernförderlichen Arbeitsgestaltung ' beziehungsweise der, lernförderlichen Arbeitsorganisation “ wird im Folgenden eine große Rolle spielen. Dies ist sicherlich nur ein Aspekt der Arbeitsgestaltung, aber eben nicht irgendeiner. Die Frage, inwieweit die Gestaltung der Arbeit dazu beitragen kann, dass Menschen ihre Kenntnisse und Fähigkeiten über lange Zeiträume hinweg erhalten oder sogar steigern können, beschäftigt die Arbeitspsychologie seit mindestens einem halben Jahrhundert. Zugleich ist diese Frage für die Zukunft - angesichts länger werdender Erwerbsbiografien in Kontext des demografischen Wandels - von herausragender Bedeutung.

Die Darstellung wird sich an drei Phasen der Entwicklung orientieren: Konzepte der Humanisierung des Arbeitslebens aus den Siebziger- bis Neunzigerjahren stehen am Anfang. Eine zweite Phase von Forschungs- und Entwicklungsarbeiten unter dem Motto ,Lernen im Prozess der Arbeit (LiPA) ${ }^{`}$ kennzeichnet die Zeit unmittelbar nach der Jahrtausendwende. Eine gerade entstehende Forschungslinie stellt das arbeitsimmanente Lernen in einen innovationspolitischen Kontext.

\section{Humanisierung des Arbeitslebens}

Im Jahre 1974 richtete das damalige Bundesministerium für Forschung und Technologie der sozialliberalen Koalitionsregierung das Forschungsprogramm ,Humanisierung des Arbeitslebens ' ein. Damit gab es in Deutschland erstmalig ein nationales Forschungs- und Entwicklungsprogramm für die Arbeitsforschung und -gestaltung (Bieneck 2009).

Dieses Programm steht auch prototypisch für den Zeitgeist des, sozialdemokratischen Jahrzehnts' der Siebzigerjahre. Eine fortschreitende Demokratisierung der Gesellschaft „Mehr Demokratie wagen!“ - und erweiterte Partizipationsmöglichkeiten der Bürger waren Leitthemen. Demokratie und Partizipation sollten sich nicht nur auf die politischen, gesellschaftlichen und kulturellen Bereiche beziehen, sondern auch auf Wirtschaft und Arbeitswelt. Vor diesem Hintergrund ist auch die Verabschiedung des Mitbestimmungsgesetzes im Jahr 1976 zu verstehen.

Der Anspruch, die Wirtschafts- und Arbeitswelt zu demokratisieren, beschränkte sich dabei keineswegs auf Deutschland. Insbesondere die skandinavischen Länder verfolgten, den Traditionen ihrer gesellschaftlichen und wirtschaftlichen Kulturen folgend, ganz ähnliche Ansätze; als Beispiel sei das Programm ,Industrielle Demokratie“ in Norwegen genannt (Emery und Thorsrud 1976).

Im Umfeld des Programms ,Humanisierung des Arbeitslebens' (HdA) und seiner - bis heute andauernden - Nachfolgeprogramme wurden wichtige Prinzipien der Arbeitsgestaltung formuliert, die immer noch Gültigkeit besitzen. 
Auf einer ganz grundlegenden Ebene wurde das Konzept des soziotechnischen Systems (Emery und Trist 1960) - das in Ansätzen schon in den Fünfzigerjahren in Großbritannien am Tavistock-Institut entwickelt worden war (Trist und Bamforth 1951) - aufgegriffen und zu einer soziotechnischen Gestaltungsphilosophie weiterentwickelt.

Im Kern besagt dieses Konzept, dass industrielle Arbeitssysteme aus den Teilsystemen ,Mensch“, ,Organisation“ und ,Technik' bestehen, die in der Gestaltung gemeinsam und in ihren wechselseitigen Abhängigkeiten betrachtet werden müssen. Dies erscheint uns heute einigermaßen offensichtlich, stellte aber eine völlig neue Perspektive dar in einer Zeit, die von einer quasi naturgesetzlichen Kraft des technologischen Fortschritts überzeugt war. Der überraschende Befund von Trist und Bamforth im Jahre 1951 bestand darin, dass eine fortgeschrittene Automatisierung - im Bereich des Steinkohlebergbaus - nicht, wie selbstverständlich erwartet, auch zu Rationalisierung und besserer Wirtschaftlichkeit geführt hatte. Als Grund dafür identifizierten die Autoren, dass durch die damals neue Technik der Kohlegewinnung etablierte und effiziente Formen der Arbeitsorganisation zwischen den Bergleuten zerbrochen worden waren.

Auch heutzutage ist es keineswegs selbstverständlich, dass Belange des Menschen und der Organisation bei Technikentwicklung und -einführung systematisch und effektiv berücksichtigt würden. Das Prinzip der soziotechnischen Gestaltung harrt immer noch seiner breiten und nachhaltigen Implementierung.

Ein weiteres wichtiges Konzept aus dem Umfeld der Humanisierung des Arbeitslebens ist die vollständige Tätigkeit oder vollständige Handlung (Hacker 1973; Volpert 1974). Winfried Hacker unterscheidet zwei Dimensionen der Vollständigkeit von Handlungen oder Aufgaben. Die sequenzielle Vollständigkeit bezieht sich darauf, dass zu den Aufgaben eines Beschäftigten nicht nur ausführende, sondern auch organisierende, planende und kontrollierende Operationen gehören. Hierarchisch vollständig sind die Aufgaben eines Arbeitsplatzes dann, wenn zu ihrer Erfüllung geistig mehr (z. B. Problemlösen) und weniger (z. B. Routineaufgaben) anspruchsvolle Operationen in einem angemessenen Verhältnis erforderlich sind.

Es wurde schon damals deutlich, dass vollständige Handlungsstrukturen die Basis einer lernförderlichen Arbeitsorganisation sind. Nur durch kognitiv anspruchsvolle Handlungselemente werden Kenntnisse und Fähigkeiten immer wieder gefordert und in ihrer Entwicklung gefördert. Zugleich wurde deutlich, dass soziotechnische Gestaltungsprinzipien aus zwei Gründen wichtige Instrumente der Umsetzung lernförderlicher Arbeitssysteme sind. Einerseits ist die Vollständigkeit von Aufgabenstrukturen eine direkte Konsequenz der jeweils herrschenden Organisationsphilosophien (z. B. mehr oder weniger ausgeprägte Arbeitsteilung). Andererseits setzt Technik Rahmenbedingungen für die Organisation, sie stellt in gewisser Hinsicht sogar ,geronnene Organisation “ dar, man denke als Beispiel nur an die Fließbandmontage. Vollständige Aufgabenstrukturen werden sich also nur durch systemisch betrachtete Technik- und Organisationsgestaltung herbeiführen lassen. Diese Gedanken finden sich im Ansatz schon in einem ,Leitfaden zur qualifizierenden Arbeitsgestaltung', der im Kontext des Programms HdA publiziert wurde (Duell und Frei 1986). 
Ein drittes Prinzip aus dieser Zeit, das ebenfalls wichtig ist für lernförderliche Arbeitsgestaltung, wurde von Eberhard Ulich 1977 als ,differenziell-dynamische Arbeitsorganisation“ beschrieben (Ulich 1978). Der differenzielle Aspekt besteht darin, dass in einem Arbeitssystem Aufgaben für Menschen mit unterschiedlichen Fähigkeiten und Leistungsvoraussetzungen vorhanden sein sollten. Dies ist von hoher aktueller Bedeutung im Hinblick auf altersgemischte Teams im Kontext des demografischen Wandels. Ein nur differenziell gestaltete Arbeitssystem könnte allerdings dazu führen, dass die Mitarbeiter in ihren Fähigkeiten ,eingefroren “ werden und keine Entwicklungsanreize erkennen können. Deshalb besteht der dynamische Aspekt darin, dass durch systematischen Aufgabenwechsel und Aufgabenbereicherung eben diese Entwicklungsimpulse gegeben werden.

Wichtige Gestaltungsfelder im HdA-Kontext waren die industrielle Fertigung und Montage. Ein prominentes Thema im Fertigungsbereich bezog sich auf die menschengerechte und lernförderliche Gestaltung der Arbeit mit CNC-Werkzeugmaschinen, einer damals recht neuen Technologie. Gestaltungsansätze bezogen sich sowohl auf die Arbeitsorganisation - Werkstattprogrammierung von Werkzeugmaschinen - als auch auf die Gestaltung der CNC-Steuerungen und -Programmiersysteme selbst im Hinblick auf eine intuitive und werkstattgerechte Mensch-Maschine-Schnittstelle (Blum und Hartmann 1988; Hartmann et al. 1994; Henning et al. 1994).

Im Folgenden soll anhand eines Beispiels aus der Montage im Maschinenbau eine praktische Anwendung der oben beschriebenen Prinzipien illustriert werden. Dieses Projekt war nicht im Programm HdA gefördert worden, sondern wurde vom Anwenderunternehmen selbst finanziert. Es wurden allerdings, wie gesagt, etliche HdA-Prinzipien in der Projektplanung und -durchführung berücksichtigt.

Abbildung 1 zeigt als Prinzipskizze die Auslegung eines Montagesystems für Traktoren. Dieses Montagesystem sollte speziell für Gruppenarbeit tauglich sein (Hartmann 1995).

Die kleinen, unbeschrifteten Rechtecke stellen Schubpaletten dar. Diese Paletten sind 24 Quadratmeter groß. Sie sind bündig mit dem Hallenboden und werden mit einem Reibradantrieb langsam durch die Montagehalle bewegt. Auf jeder Palette befindet sich eine hydraulische Vorrichtung, auf der der Traktor während der Montage gelagert ist. Dadurch können die Werker die Arbeitshöhe flexibel einstellen.

Die Arbeitsbereiche der Gruppen sind durch die Quadrate oberhalb und unterhalb der Paletten gekennzeichnet; zu den Arbeitsbereichen gehören immer auch die Paletten, die gerade im Bereich der Gruppe sind (in der Skizze die direkt angrenzenden gelben Rechtecke).

Aus der Skizze ist erkennbar, dass zu den jeweiligen taktgebundenen Montagebereichen auf den Paletten immer auch nicht oder nicht unmittelbar taktgebundene Bereiche gehören. Dies sind insbesondere Vormontagebereiche (subassembly). So gehört etwa zu den Aufgaben der Vorderachsgruppe nicht nur die - taktgebundene - Montage der Vorderachse an den Traktor auf der Schubpalette, sondern auch die Vormontage der Achse im angrenzenden, nicht taktgebundenen Bereich. 


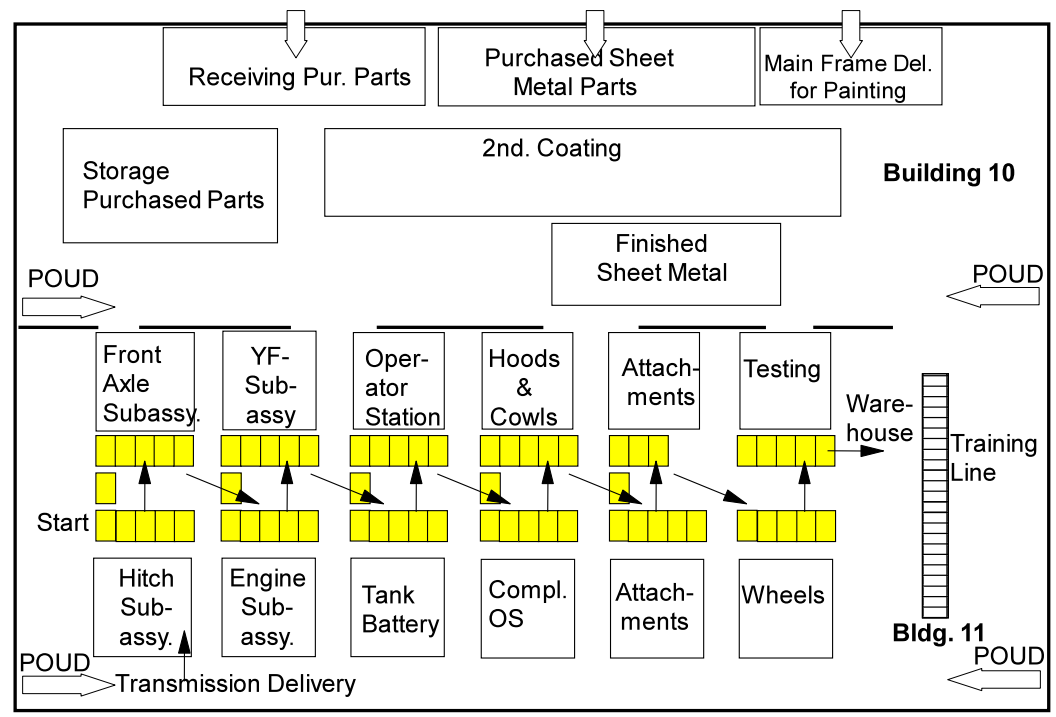

Abb. 1 Montagesystem für landwirtschaftliche Traktoren. POUD: Point of use delivery; YF: yellow frame, Auflage der Motorhaube; OS: Operator Station, Kabine (Farbabbildung online), Quelle: Hartmann E.A. (1995): Human-Oriented Technology, Tokyo

Dadurch entstehen unterschiedliche Aufgabenstrukturen mit unterschiedlichen Anforderungen, im Sinne der differenziellen Arbeitsgestaltung. Der dynamische Aspekt wurde dadurch realisiert, dass auf Polyvalenz der Mitarbeiter - möglichst jeder soll mehrere Arbeitsplätze beherrschen - großer Wert gelegt wurde; dies wurde auch in Kennzahlen für die einzelnen Montagebereiche abgebildet.

Im Sinne der hierarchischen und sequenziellen Vollständigkeit wurden Aufgaben der Arbeitsplanung und -koordination - innerhalb der Gruppe und darüber hinaus mit den angrenzenden Gruppen - in das Aufgabenspektrum der Montagegruppen integriert. Dies bezieht sich auch auf die kontinuierliche Verbesserung des Montagesystems.

Die soziotechnische Gestaltung zeigt sich schließlich darin, dass das ganze technische Layout - vom Montagesystem selbst bis zu den vor Ort vorhandenen Gruppenräumen auf eine bestimmte Organisationsphilosophie - Gruppenarbeit - ausgerichtet wurde. Diese Organisationsphilosophie berücksichtigt wiederum - im Sinne vollständiger Aufgabenstrukturen - menschliche Bedürfnisse.

Dieses Montagesystem ist bis heute nach derselben Philosophie sehr erfolgreich in Betrieb und gilt immer noch als eine der modernsten Traktorenmontagen der Welt.

Solche umfassenden und nachhaltigen Lösungen waren allerdings unter den Ergebnissen der HdA-Projekte nicht sehr häufig zu finden. Ein Grund dafür mag in der stark gesellschaftspolitischen Ausrichtung des Programms gelegen haben, wodurch Bezüge zu Unternehmensstrategien oftmals unklar oder fragil blieben. 
Davon unbenommen hat die Forschung und Entwicklung im Kontext des HdAProgramms und seiner Nachfolgeprogramme grundlegende Erkenntnisse und Methoden hervorgebracht, die bis heute nichts von ihrer Bedeutung und Gültigkeit verloren haben.

\section{Lernen im Prozess der Arbeit}

Unmittelbar nach der Jahrtausendwende wurde das Thema lernförderlicher Arbeitsorganisation in einem anderen Kontext aufgegriffen, im BMBF-Programm ,Lernkultur Kompetenzentwicklung ' (Erpenbeck und Sauer 2001; Hartmann und von Rosenstiel 2004).

Während das HdA-Programm von einer gesellschaftspolitischen Stoßrichtung gekennzeichnet war, bezog sich ,Lernkultur Kompetenzentwicklung ' auf bildungspolitische Ziele. Ursprung und Motivation des Programms lagen darin, dass nach Möglichkeiten des lebenslangen Lernens jenseits klassischer, formaler, an Bildungseinrichtungen gebundener Weiterbildung gesucht wurde (Staudt und Kriegesmann 1999). Formen des informellen Lernens wurde verstärkte Aufmerksamkeit zugewendet. Damit war die Hoffnung verbunden, dass informelles Lernen - ergänzend zum Lernen in (Weiter-) Bildungseinrichtungen - neue Potenziale entfalten könnte.

$\mathrm{Zu}$ diesen Potenzialen gehört zum ersten, dass bestimmte Fähigkeiten-Kompetenzenbesonders gut in informellen Lernsituationen erworben werden. Kompetenzen im von John Erpenbeck vorgeschlagenen Sinn sind Selbstorganisationsdispositionen. Während sich Qualifikationen auf bestimmte Anforderungssituationen beziehen - Arbeitsplätze oder Tätigkeitsfelder - sind Kompetenzen diejenigen Fähigkeiten, die es uns ermöglichen, in unbestimmten, neuen, unstrukturierten Situationen handlungsfähig zu sein (Erpenbeck und Sauer 2001). Solche Kompetenzen entwickeln sich vermutlich insbesondere in realen Handlungssituationen.

Zweitens reduziert informelles Lernen das Transferproblem: Lern- und Anwendungskontext sind identisch. Drittens erweitert informelles Lernen den Zugang zur Weiterbildung, sofern informell erworbene Kompetenzen erfasst, validiert und formal anerkannt werden können (Colardyn und Bjørnåvold 2004). Viertens und schließlich ist informelles Lernen effizient: Die Lernzeit addiert sich nicht zur Arbeitszeit, vielmehr sind - lernförderliche Handlungskontexte vorausgesetzt - Arbeitszeiten zugleich Lernzeiten.

Abbildung 2 zeigt sie Grundstruktur des Forschungs- und Entwicklungsprogramms ,Lernkultur Kompetenzentwicklung‘. Das Lernen im Prozess der Arbeit steht hier neben anderen informellen Lernformen - im sozialen Umfeld und mit digitalen Medien - sowie formalem bzw. non-formalem ${ }^{1}$ Lernen in Weiterbildungseinrichtungen.

\footnotetext{
${ }^{1}$ Der Unterschied zwischen formalem und non-formalem Lernen besteht darin, dass nur ersteres mit breit anerkannten Zertifikaten belegt und ausgewiesen wird (z. B. Hochschulabschluss). Gemeinsam ist beiden Lernformen, dass sie in expliziten Lernumgebungen - Bildungseinrichtungen - situiert sind, dadurch unterscheiden sich beide vom informellen Lernen. Im Fall des non-formalen Lernens sind dies beispielsweise Weiterbildungseinrichtungen.
} 
Abb. 2 Struktur des

Forschungs- und

Entwicklungsprogramms

,Lernkultur

Kompetenzentwicklung',

Quelle: John Erpenbeck,

Johannes Sauer (2001): Das

Forschungs- und

Entwicklungsprogramm

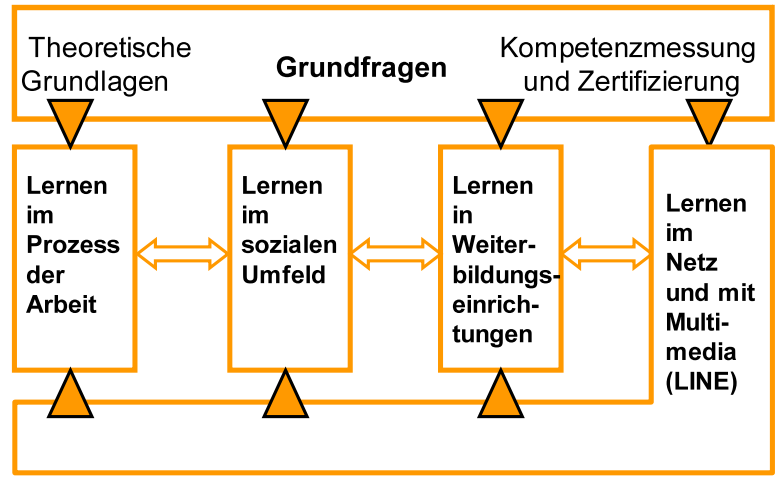

Das Programm ,Lernkultur Kompetenzentwicklung‘ stimulierte im Bereich der Förderlinie ,Lernen im Prozess der Arbeit (LiPA)` vielfältige Forschungsarbeiten. Besonders hervorzuheben sind diejenigen, die die Entwicklung von empirisch bestimmbaren Maßen und Kennzahlen der Lernförderlichkeit von Arbeitssituationen gewidmet waren (Bergmann et al. 2004; Frieling et al. 2006). Die Ergebnisse dieser Forschungen reichen an dieser Stelle weit über die Erkenntnisse aus HdA hinaus. Sie stellen zugleich Grundlagen zu Verfügung, die für eine ,ingenieursmäßige‘ Gestaltung industrieller Arbeitssysteme genutzt werden kann. Darauf wird zurückzukommen sein.

Schwächen der Programmlinie LiPA bestanden dahingehend, dass Forschung einerseits und Entwicklung sowie Anwendung andererseits auseinanderfielen. Während die Forschung sehr präzise den Beitrag der Arbeitsgestaltung zum Lernen in der Arbeit adressierte, wurde in den Entwicklungsprojekten die tatsächliche Gestaltung von Arbeitssystemen kaum betrieben. Damit korrespondierend beteiligten sich aus den Unternehmen vor allem Personal- und Weiterbildungsabteilungen. Für eine substanzielle Arbeitsgestaltung wäre allerdings die Einbindung von Akteuren wie technische Planung, Industrial Engineering oder auch IT-Engineering notwendig gewesen (Wessels 2009).

Als zentrales Verdienst von LiPA für die lernförderliche Arbeitsgestaltung bleibt die Entwicklung fundierter und perspektivisch auch praktisch anwendbarer Kennzahlen der Lernförderlichkeit von Arbeitssituationen und -systemen.

\section{Innovative Arbeitsplätze - Arbeit und Innovationsfähigkeit}

In der jüngsten Zeit entwickelt sich eine neue Perspektive auf die lernförderliche Arbeitsgestaltung, die weder gesellschafts- noch bildungspolitisch, sondern innovationspolitisch ausgerichtet ist (Lorenz und Valeyre 2005; OECD 2010; Hartmann und Garibaldo 2011; CEDEFOP 2012; Hartmann et al. 2014).

Diese Beiträge weisen darauf hin, dass für die Analyse der Innovationsfähigkeit von Unternehmen die Betrachtung von FuE-Aufwendungen und Anteilen Hochqualifizierter in der Belegschaft zu kurz greift. Für eine substanzielle Innovationsfähigkeit ist es vielmehr 
erforderlich, dass quer durch die Belegschaften und die betrieblichen Funktionsbereiche hoch entwickelte Kompetenzen vorhanden sind. Dies ist die zentrale Voraussetzung für eine beidseitige Kommunikation zwischen Produktion, Konstruktion, Entwicklung und Forschung. Auch Prozessinnovationen durch kontinuierliche Verbesserungen erfordern Kompetenzen in allen betroffenen Bereichen. Diese Kompetenzen werden - ganz im Sinne von LiPA - nicht nur, vielleicht auch nicht vorrangig durch formale (Weiter-)Bildung aufgebaut. Besonders bedeutsam ist vielmehr das Lernen in der Arbeit, ermöglicht durch lernförderliche Arbeitsorganisation (OECD 2010).

Daher verwundert es nicht, dass sich zwischen Kennwerten der lernförderlichen Arbeitsorganisation ${ }^{2}$ und der Innovationsleistung - gemessen z. B. in Innovatorenquoten bedeutsame Zusammenhänge auf Länderebene zeigen lassen (Lorenz und Valeyre 2005; OECD 2010; CEDEFOP 2012). Diese Zusammenhänge sind eher stärker als sie zwischen dem Anteil Hochqualifizierter (Akademiker) an den Belegschaften, einem in der Innovationsindikatorik sehr gängigen Prädiktor der Innovationsleistung (CEDEFOP 2012).

Abbildung 3 zeigt Daten des iit-Innovationsfähigkeitsindikators ${ }^{3}$ zur lernförderlichen Arbeitsorganisation für Deutschland im Vergleich zu den Durchschnittswerten europäischer Länder (EU27 und Norwegen; Hartmann et al. 2014). Es werden zwei Dimensionen der lernförderlichen Arbeitsorganisation unterschieden Handlungsspielraum und Aufgabenkomplexität. Diese Dimensionen finden sich in sehr ähnlicher Form auch in den oben dargestellten Indikatorensystemen zur Lernförderlichkeit von Arbeitssituationen (Bergmann et al. 2004; Frieling et al. 2006). Die Aufgabenkomplexität kann dabei durchaus als ein Hinweis auf die (hierarchische) Vollständigkeit von Aufgaben gewertet werden (Hacker 1973).

Wie aus Abb. 3 ersichtlich, liegen die Werte für Deutschland hinsichtlich des Handlungsspielraum niedriger, hinsichtlich der Aufgabenkomplexität höher als der europäische Durchschnitt. Dazu ist anzumerken, dass die Aufgabenkomplexität offensichtlich ein etwas bedeutsamerer Indikator der Innovationsfähigkeit als der Handlungsspielraum ist (CEDEFOP 2012).

Im iit-Indikator ${ }^{4}$ wird die lernförderliche Arbeitsorganisation eingebettet in ein theoretisch fundiertes und empirisch belegbares Konzept der Innovationsfähigkeit. Dieses Konzept unterscheidet vier Säulen der Innovationsfähigkeit. Dazu gehören erstens das für die Unternehmen verfügbare spezialisierte Fachwissen (Humankapital), zweitens die Vielfalt spezialisierter Wissensbestände und die Fähigkeit, diese in der Erzeugung komplexer Produkte zu verknüpfen (Komplexitätskapital), drittens betriebliche Strukturen, die die Erzeugung von Wissen aus FuE, aber auch in den unmittelbaren Arbeitsprozessen ermöglichen (Strukturkapital), und schließlich die Vernetzung mit externen Wissensträgern wie Forschungs- und Bildungseinrichtungen (Beziehungskapital).

\footnotetext{
${ }^{2}$ Die Autoren greifen hier auf Daten der Europäischen Befragung zu Arbeitsbedingungen zurück; European Work Condition Survey (EWCS), http://eurofound.europa.eu/working/surveys/.

${ }^{3}$ Auch diese Daten basieren auf dem EWCS.

${ }^{4}$ http://www.iit-berlin.de/de/indikator.
} 
Unterteilung des Unterindikators "Lernförderliche Arbeitsorganisation" der Hauptsäule

"Strukturkapital": Vergleich Deutschland und EU (inkl. Norwegen)

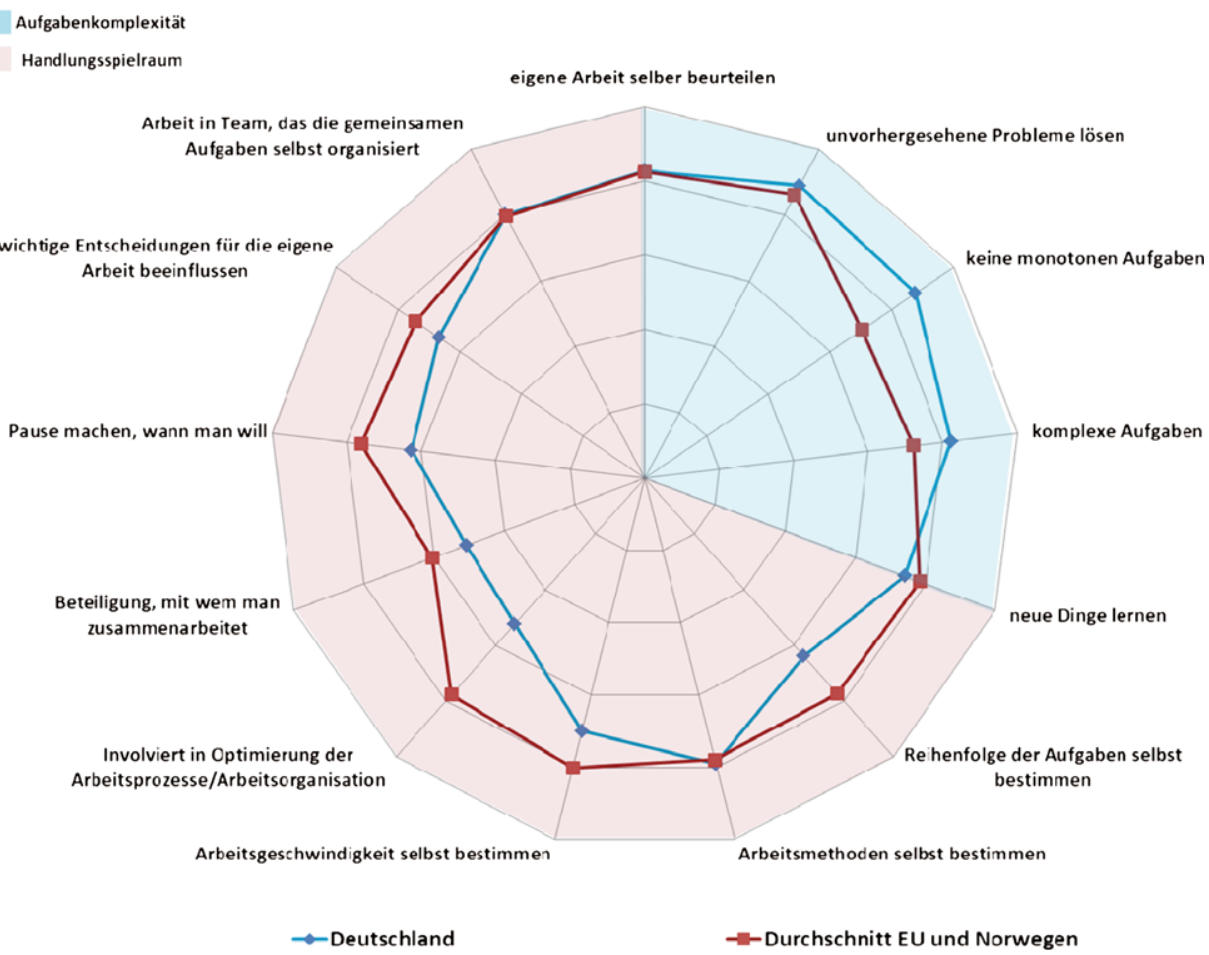

Abb. 3 Lernförderliche Arbeitsorganisation als Element des Strukturkapitals, iit-Innovationsfähigkeitsindikator, Quelle: Institut für Innovation + Technik (iit)

Die lernförderliche Arbeitsorganisation wird hier also - als wesentliches Element des Strukturkapitals - in ein breites Konzept der Innovationsfähigkeit von Unternehmen und letztlich Volkswirtschaften integriert (Hartmann et al. 2014).

\section{Fazit und Ausblick}

Aus den drei Entwicklungsphasen der Forschung zur lernförderlichen Arbeitsorganisation liegen bedeutsame Erträge vor. HdA erbrachte die theoretischen und methodischen Grundlagen, die die Analyse und Gestaltung von lernförderlichen Arbeitssystemen bis heute prägen. LiPA ergänzte Methoden zur Bestimmung konkreter Kennzahlen für die Lernförderlichkeit von Arbeitssituationen. Jüngere Arbeiten zu ,innovativen Arbeitsplätzen “ machen schließlich deutlich, dass die Frage lernförderlicher Arbeitssysteme keine Marginalie darstellt, sondern vielmehr einen zentralen Faktor der Innovationsfähigkeit und damit der technologischen und wirtschaftlichen Wettbewerbsfähigkeit. 
Für die Zukunft sollen zwei Herausforderungen benannt werden. Erstens wird es darum gehen, die Erkenntnisse - etwa aus LiPA - zur Analyse und Beschreibung lernförderlicher Arbeit zu nutzen für die tatsächliche Gestaltung von Arbeitssystemen in der Breite der Industrie. Dieser Aufgabe widmet sich das vom Bundesministerium für Bildung und Forschung geförderte Projekt, Engineering und Mainstreaming lernförderlicher industrieller Arbeitssysteme für die Industrie 4.0 (ELIAS) ${ }^{6}$, das kürzlich (Ende 2013) seine Arbeit aufgenommen hat. ${ }^{5}$ Neben Unternehmen und Forschungseinrichtungen (insbesondere der RWTH Aachen) ist die Deutsche MTM-Vereinigung als bedeutende Organisation des Industrial Engineering am Projekt beteiligt. In Deutschland arbeiten ungefähr drei Millionen Menschen in Arbeitssystemen, die nach MTM-Methodik gestaltet wurden. Dies spricht für erhebliche Transferpotenziale und Hebelwirkungen für die zukünftigen Projektergebnisse.

Schließlich solle ein Problem benannt werden, dass schon vor 30 Jahren von Lisanne Bainbridge erkannt und bis heute nicht gelöst wurde (Bainbridge 1983). Die von ihr beschriebenen ,Ironies of Automation“ - Automatisierungsdilemmata - stellen eine erhebliche Hürde für lernförderliche Arbeitsgestaltung im Kontext automatisierter Arbeitssysteme dar.

Das Kerndilemma - die ,Ironie“ der Automatisierung - lässt sich wie folgt beschreiben. Die Automatisierung von Prozessen führt häufig dazu, dass Menschen diese automatisch ablaufenden Prozesse nur noch überwachen. In seltenen Fällen ist das automatische System allerdings überfordert, und der Mensch muss eingreifen. Das Problem besteht nun darin, dass der menschliche Operateur aus mehreren Gründen schlecht in der Lage ist, diese Situationen zu bewältigen. Dies liegt zunächst am besonderen Anforderungsgehalt der Situation: Der Automat wird tendenziell dann die Situation nicht mehr beherrschen, wenn die Situation besonders anspruchsvoll ist. Zweitens ist der Mensch, weil er die Situation nicht selbst herbeigeführt hat, kaum , aktuell im Bilde“ und wenig in der Lage, die Situation schnell zu analysieren und Handlungsoptionen abzuleiten. Drittens wird auch die grundsätzliche Fähigkeit des Menschen, das automatisierte System und die Umgebung insgesamt zu verstehen, mit der Zeit abnehmen, je weniger er aktiv in die Systemsteuerung eingreifen muss. Es entsteht eine ,ironische ' Problemlage: Der menschliche ,Überwacher ‘ ist gerade wegen der Automatisierung zunehmend weniger in der Lage, seiner Überwachungstätigkeit gegenüber dem automatisierten System nachzugehen.

Cyberphysikalische Systeme könnten hier Abhilfe schaffen durch neue Möglichkeiten der Erfassung, Aufbereitung und Visualisierung von Prozessdaten, die es dem Nutzer ermöglichen, ,im Bilde zu bleiben“. Andere Autoren dieses Bandes - insbesondere Andreas Lüdtke und Bernd Kärcher - stellen diese Möglichkeiten im Detail dar.

Open Access This chapter is distributed under the terms of the Creative Commons Attribution Noncommercial License, which permits any noncommercial use, distribution, and reproduction in any medium, provided the original author(s) and source are credited.

\footnotetext{
${ }^{5}$ http://projekte.fir.de/elias/.
} 


\section{Literaturverzeichnis}

Bainbridge, L. (1983). Ironies of automation. Automatica, 19(6), 775-779.

Bergmann, B., Richter, F., Pohlandt, A., Pietrzyk, U., Eisfeldt, D., Hermet, V., \& Oschmann, D. (2004). Arbeiten und Lernen. Münster: Waxmann.

Bieneck, H.-J. (2009). Humanisierung des Arbeitslebens - ein sozial- und forschungspolitisches Lehrstück. Zeitschrift für Arbeitswissenschaft, 63(2), 112-115.

Blum, U., \& Hartmann, E. A. (1988). Facharbeiterorientierte CNC-Steuerungs- und -Vernetzungskonzepte. Werkstatt und Betrieb, 121, 441-445.

CEDEFOP (2012). Learning and innovation in enterprises. Research Paper No. 27. Luxembourg: Publications Office of the European Union.

Colardyn, D., \& Bjørnåvold, J. (2004). Validation of formal, non-formal and informal learning: policy and practices in EU member states. European Journal of Education, 39(1).

Duell, W., \& Frei, F. (1986). Leitfaden für qualifizierende Arbeitsgestaltung. Köln: Verlag TÜV Rheinland.

Emery, F. E., \& Thorsrud, E. (1976). Democracy at work. The report of the Norwegian Industrial Democracy Programme. Leiden: Nijhoff.

Emery, F. E., \& Trist, E. L. (1960). Socio-technical systems. In C. W. Churchman \& M. Verhulst (Hrsg.), Management. Sciences, models, and technics (S. 83-97). New York: Pergamon Press.

Erpenbeck, J., \& Sauer, J. (2001). Das Forschungs- und Entwicklungsprogramm „Lernkultur Kompetenzentwicklung“. QUEM-report-Schriften zur beruflichen Weiterbildung, 67, 9-66.

Frieling, E., Bernard, H., Bigalk, D., \& Müller, R. (2006). Lernen durch Arbeit - Entwicklung eines Verfahrens zur Bestimmung der Lernmöglichkeiten am Arbeitsplatz. Münster: Waxmann.

Hacker, W. (1973). Allgemeine Arbeits- und Ingenieurpsychologie. Psychische Struktur und Regulation von Arbeitstätigkeiten. Berlin: VEB Deutscher Verlag der Wissenschaften.

Hartmann, E. A. (1995). Specifying requirements for human-oriented technology in tractor manufacturing. In Proceedings of the international symposium on human oriented manufacturing systems. Tokyo: Waseda University.

Hartmann, E. A., \& Garibaldo, F. (2011). What's going on out there? Designing work systems for learning in real life. In S. Jeschke, I. Isenhardt, F. Hees, \& S. Trantow (Hrsg.), Enabling innovation: innovative capability - German and international views. Berlin: Springer.

Hartmann, E. A., \& von Rosenstiel, L. (2004). Infrastrukturelle Rahmenbedingungen der Kompetenzentwicklung. In Arbeitsgemeinschaft Betriebliche Weiterbildungsforschung (Hrsg.), Kompetenzentwicklung 2004. Münster: Waxmann.

Hartmann, E. A., Fuchs-Frohnhofen, P., \& Brandt, D. (1994). Designing CNC-machine tools to fit the skilled workers at the individual workplace and in group work. In Proceedings of the international federation of automatic control (IFAC) conference: integrated systems engineering, Baden-Baden, 27.-29.09.1994.

Hartmann, E. A., von Engelhardt, S., Hering, M., Wangler, L., \& Birner, N. (2014). Der iit-Innovationsfähigkeitsindikator - Ein neuer Blick auf die Voraussetzungen von Innovationen, iit Perspektive $N r$. 16, online: http://www.iit-berlin.de/de/publikationen/der-iitinnovationsfaehigkeitsindikator.

Henning, K., Volkholz, V., Risch, W., \& Hacker, W. (Hrsg.) (1994). Moderne LernZeiten. Berlin: Springer.

Locke, R. M. \& Wellhausen, R. L. (Eds.) (2014). Production in the innovation economy. Cambridge: MIT Press.

Lorenz, E., \& Valeyre, A. (2005). Organisational innovation, HRM and labour market structure: a comparison of the EU15. Journal of Industrial Relations, 47, 424-442.

OECD (2010). Innovative workplaces: making better use of skills within organisations, OECD Publishing. http://dx.doi.org/9789264095687-en. 
Staudt, E., \& Kriegesmann, B. (1999). Weiterbildung: Ein Mythos zerbricht. In E. Staudt (Hrsg.), Berichte aus der angewandten Innovationsforschung (Bd. 178). Bochum

Promotorengruppe Kommunikation der Forschungsunion Wirtschaft - Wissenschaft (Hrsg.) (2013). Deutschlands Zukunft als Produktionsstandort sichern - Umsetzungsempfehlungen für das Zukunftsprojekt Industrie 4.0. Abschlussbericht des Arbeitskreises Industrie 4.0. Online: http://www.bmbf.de/pubRD/Umsetzungsempfehlungen_Industrie4_0.pdf.

Trist, E. L., \& Bamforth, K. W. (1951). Some social and psychological consequences of the Longwall method of coal-getting. Human Relations, 4, 3-38.

Ulich, E. (1978). Über das Prinzip der differentiellen Arbeitsgestaltung. Industrielle Organisation. 47, 566-568.

Volpert, W. (1974). Handlungsstrukturanalyse als Beitrag zur Qualifikationsforschung. Köln: PahlRugenstein.

Wessels, J. (2009). Nationale und internationale Wissensbestände zum Lernen im Prozess der Arbeit (LiPA), Expertise im Rahmen des Internationalen Monitorings zum Forschungs- und Entwicklungsprogramm „Arbeiten-Lernen-Kompetenzen entwickeln - Innovationsfähigkeit in einer modernen Arbeitswelt“, online: http://www.internationalmonitoring.com/fileadmin/Downloads/ Experten/Expertisen/Expertisen_neu/Expertise_Wessels.pdf. 